\title{
The Association Between Delayed Graft Function and Renal Resistive Index in Kidney Transplant Recipients
}

\author{
Serkan Bakirdogen ${ }^{1}$, Hasan Anil Kurt ${ }^{2}$, Fatih Kamış ${ }^{3}$, Sibel Bek ${ }^{4}$, Aysegul Erbayraktar ${ }^{3}$ \\ 1. Nephrology Department, Canakkale 18 Mart University, Canakkale, TUR 2. Urology, Canakkale Onsekiz Mart \\ University, School of Medicine, Canakkale, TUR 3. Internal Medicine, Canakkale Onsekiz Mart University, School of \\ Medicine, Canakkale, TUR 4. Nephrology, Kocaeli University Hospital, Kocaeli, TUR
}

Corresponding author: Serkan Bakirdogen, sbdogen@comu.edu.tr

\section{Abstract \\ Background and objectives}

Delayed graft function (DGF) may increase the risk for kidney graft dysfunction. Renal resistive index (RRI) in Doppler ultrasonography is useful in measuring blood flow changes in kidneys which is indicative of tubulointerstitial damage. Most of the diseases in DGF etiology are related to tubulointerstitium and arteries of the kidneys. In this study, we investigated whether there is a relationship between delayed graft function and renal resistive index in kidney transplant recipients (KTR).

\section{Materials and methods}

Patients who underwent kidney transplantation were included in this retrospective study. KTR were divided into two groups as DGF (+) and DGF (-). Comparison of RRI values of DGF (+) and DGF (-) groups according to the measurements at different times.

\section{Results}

The findings showed that both RRI measurements (post-transplant in the first week and the end of the first year) of the DGF (+) group were higher than DGF (-) group ( $\mathrm{p}=0.001$ and $\mathrm{p}=0.003$, respectively). The interaction of measurements and DGF did not have an effect on RRI ( $p>0.05)$.

\section{Conclusion}

The value of RRI in the DGF (-) group was lower than DGF (+) group in the first week after kidney transplantation.

Review began 07/19/2021 Review ended 08/15/2021 Published 08/19/2021

\section{(๑) Copyright 2021}

Bakirdogen et al. This is an open access article distributed under the terms of the Creative Commons Attribution License CC-BY 4.0., which permits unrestricted use, distribution, and reproduction in any medium, provided the original author and source are credited.
Categories: Radiology, Nephrology, Transplantation

Keywords: delayed graft function, doppler ultrasound, kidney transplant recipient, post transplantation, renal resistive index

\section{Introduction}

Delayed graft function (DGF) is defined as the need for dialysis within the first week after kidney transplantation. The etiology of DGF includes acute tubular necrosis, accelerated/acute rejection, thrombotic microangiopathy, vascular and surgical complications (ureter stenosis, urinary leakage) $[1,2]$. The effects of DGF on graft survival in the future are controversial. The prognosis may vary according to the underlying etiology. DGF may increase the risk for graft dysfunction and mortality in the late period [1,3-6].

Renal resistive index (RRI) in Doppler ultrasonography is useful in measuring blood flow changes in kidneys and showing damage to the tubulointerstitium [7]. The decrease in renal artery blood flow was associated with prolonged cold ischemia time and the development of DGF [8,9]. Most of the diseases in DGF etiology may affect the tubulointerstitium of the kidneys $[1,2]$. In this study, we investigated whether there is a relationship between delayed graft function and renal resistive index in kidney transplant recipients (KTR).

\section{Materials And Methods}

Our study was designed retrospectively. Patients who underwent renal transplantation in the organ transplant unit of our hospital between October 2015 and March 2020 were included in this study. DGF was defined as the need for dialysis within the first week after kidney transplantation [1,2]. Kidney transplant recipients were divided into two groups as DGF $(+)$ and DGF (-). Retrospective records and renal Doppler ultrasonography data of the patients were collected from the hospital. Renal artery stenosis, renal vein thrombosis, advanced aortic valve regurgitation, heart failure, acute pyelonephritis, and acute rejection were accepted as exclusion criteria for this study. This study started after the approval of the local ethics 
committee.

RRI value was determined by examining the interlobar artery in the upper, middle, and lower zones of the kidney and by the average of three different measurements. Toshiba Aplio XG Doppler ultrasonography device was used together with a convex transducer (PVT-375BT). RRI was calculated using the formula "peak systolic velocity - end-diastolic velocity/peak systolic velocity" [7]. Doppler ultrasonography was performed on the patients by the specialists in the radiology clinic of our hospital. Two (post-transplant in the first week and end of the first year) RRI measurements of each patient were recorded.

Blood was drawn from each patient in the early morning following at least eight hours of fasting. All blood samples were studied in our hospital's biochemistry laboratory. Serum creatinine and urea analyses of the patients were examined using the colorimetric method on the Roche Cobas 6000 device 501 modules. Biochemistry markers of each patient were recorded on the first day of kidney transplantation, the third month, and at the end of the first year.

The data collected from the patients in this study were transferred to the Statistical Package for the Social Sciences (SPSS) 19.0 program (IBM Corp., Armonk, NY). The demographic data of the patients, such as age and gender, were determined by descriptive statistics. Comparison of DGF (+) and DGF (-) groups on the age variable were performed using the Mann-Whitney $U$ test since they did not show normal distribution. The relationship of the patient groups concerning gender was examined by chi-square analysis. Comparison of the groups concerning RRI at post-transplant in the first week was performed by Student's t-test because it conformed to normal distribution. As the number of patients decreased, the comparison of RRI values of the groups at the end of the first post-transplant year was performed using the Mann-Whitney U test.

Bonferroni, a multiple comparison test, was conducted to investigate that there were significant differences between groups. Comparison of RRI, serum urea, and creatinine values of DGF $(+)$ and DGF (-) groups according to the measurements at different times was made using a two-factor analysis of variance (ANOVA) test.

\section{Results}

Eighty-six kidney transplant recipients (29 females, 57 males) were included in this study. The average age of the patients was $52 \pm 13.07$. Of the kidney transplant recipients, 45 (from 44 cadaveric and one living donor) were DGF (+) and 41 (from 37 cadaveric and four living donors) were DGF (-). When evaluated according to chronic kidney disease, 17 patients had diabetes mellitus, 32 had hypertension, 12 had chronic glomerulopathy, four had autosomal dominant polycystic kidney disease, three had urological causes, and 18 had an unknown etiology. Antithymocyte globulin (1-3 mg/kg/day, 2-14 days) was administered to each renal transplant recipient as induction therapy. Triple immunosuppressive therapy (tacrolimus, prednisolone, and mycophenolate mofetil) was given to each patient after transplantation.

No statistically significant difference was found between the groups regarding age $(p=0.792)$. The findings obtained in this study showed that there was no statistically significant difference in the diagnosis of DGF according to gender $(\mathrm{p}=0.937)$. Cold ischemia time was higher in DGF $(+)$ group than in DGF $(-)$ group. The result was statistically significant $(\mathrm{p}=0.002)$. The groups were found similar concerning human leukocyte antigen (HLA) mismatch $(\mathrm{p}=0.081)$.

RRI measurements of DGF (+) and DGF (-) patients in the first week after kidney transplantation and at the end of the first year were compared with each other. The estimated marginal means of RRI in both groups are shown in Figure 1. Both RRI measurements (post-transplant in the first week and end of the first year) of the DGF (+) group were higher than DGF (-) group. The results were statistically significant ( $\mathrm{p}=0.001$ and $\mathrm{p}=0.003$, respectively). The comparison of age, cold ischemia time, HLA mismatch, and RRI values of the groups after kidney transplantation are given in Table 1. 


\section{Cureus}

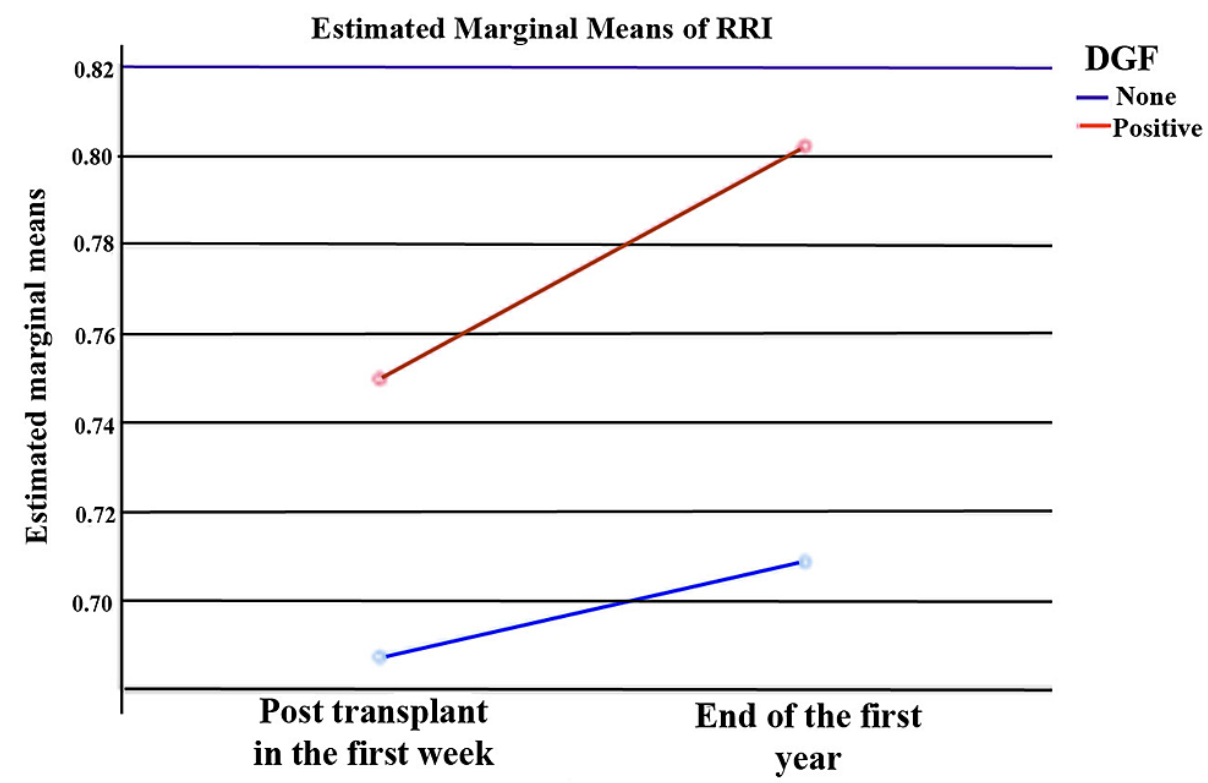

FIGURE 1: Estimated marginal means of RRI in both groups

RRI: renal resistive index.

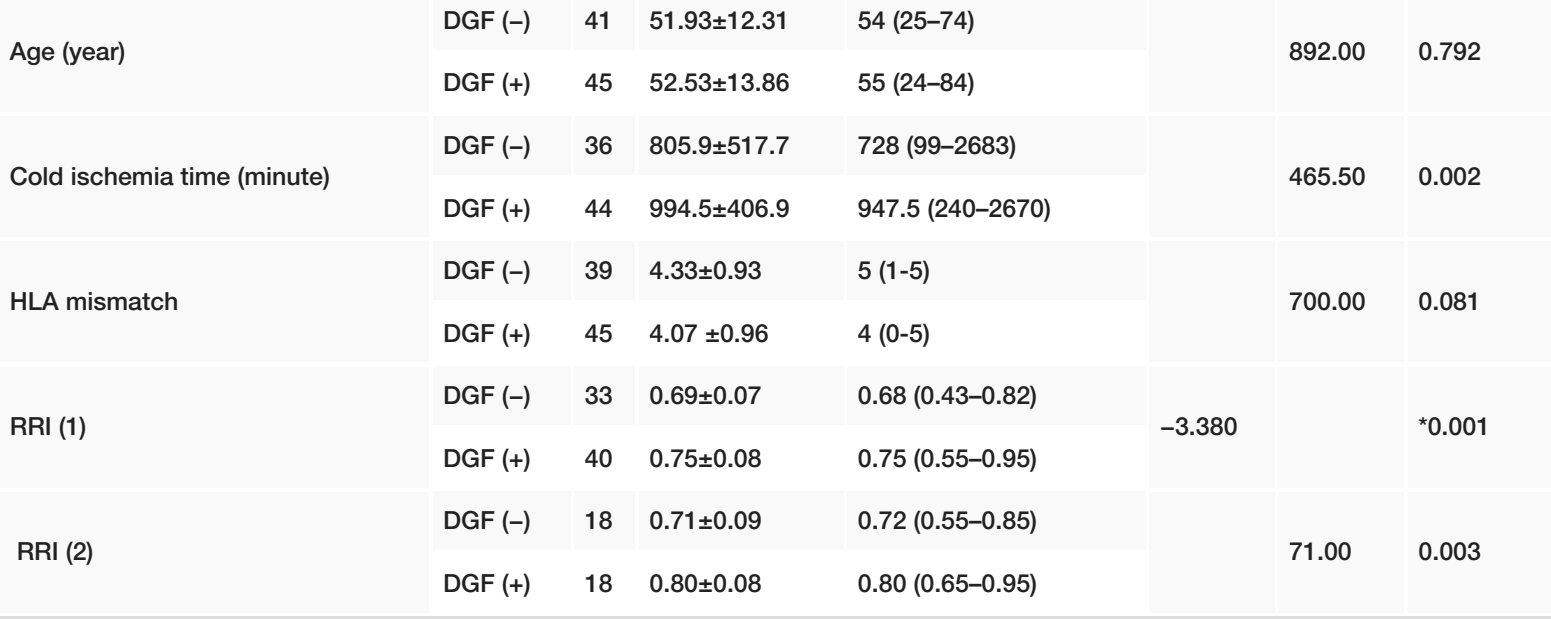

TABLE 1: The comparison of age, cold ischemia time, HLA mismatch, and RRI values of the groups after kidney transplantation

HLA: human leukocyte antigen, RRI: renal resistive index.

*The interaction of measurements.

RRI values were statistically significantly different according to the measurement $(F=5.141, p<0.05)$. RRI values were statistically significantly different according to DGF groups $(F=23.064, p<0.05)$ but it was seen that the interaction of measurements and DGF did not have a statistically significant effect on RRI ( $F=0.908$, $\mathrm{p}>0.05$ ). The comparison of the RRI values of DGF groups according to the measurement at different times is shown in Table 2. 


\section{Cureus}

\begin{tabular}{|c|c|c|c|}
\hline Source of variance & $\mathbf{F}$ & $\mathbf{p}$ & $\eta^{2}$ \\
\hline Measurement (RRI) & 5.141 & 0.025 & 0.047 \\
\hline DGF & 23.064 & 0.0001 & 0.180 \\
\hline Measurement (RRI)* DGF & 0.908 & 0.343 & 0.009 \\
\hline
\end{tabular}

TABLE 2: The comparison of the RRI values of the DGF groups according to the measurement at different times (two-factor ANOVA)

RRI: renal resistive index, DGF: delayed graft function, ANOVA: analysis of variance.

*The interaction of measurements.

Serum creatinine levels were not statistically significantly different according to the measurement $(\mathrm{F}=2.621$, $\mathrm{p}>0.05)$. Creatinine levels were statistically significantly different according to DGF groups $(F=26.95, p<0.05)$, but it was seen that the interaction of measurements and DGF did not have a statistically significant effect on serum creatinine levels $(\mathrm{F}=2.109, \mathrm{p}>0.05)$.

Serum urea levels were statistically significantly different according to the measurement $(\mathrm{F}=22.0, \mathrm{p}<0.05)$. Urea levels were statistically significantly different according to DGF groups $(\mathrm{F}=17.67, \mathrm{p}<0.05)$, but it was seen that the interaction of measurements and DGF did not have a statistically significant effect on serum urea levels ( $F=1.469, \mathrm{p}>0.05)$. Serum mean urea and creatinine levels and standard deviation values of the groups after kidney transplantation are given in Table 3 . The comparison of the mean serum urea and creatinine levels of DGF groups according to the measurement at different times is shown in Table 4 . Estimated marginal means of serum urea and creatinine levels in both groups are shown in Figure 2.

\begin{tabular}{|c|c|c|c|c|c|c|c|}
\hline \multirow{2}{*}{\multicolumn{2}{|c|}{ Measurement (after transplantation) }} & \multicolumn{3}{|c|}{ Creatinine (mg/dL) } & \multicolumn{3}{|c|}{ Urea (mg/dL) } \\
\hline & & Mean & Standard deviation & $\mathbf{N}$ & Mean & Standard deviation & $\mathbf{N}$ \\
\hline \multirow{3}{*}{ The first day } & DGF (-) & 1.78 & 0.91 & 40 & 83.92 & 36.82 & 40 \\
\hline & DGF (+) & 3.16 & 2.02 & 44 & 114.04 & 42.54 & 44 \\
\hline & Total & 2.50 & 1.73 & 84 & 99.69 & 42.47 & 84 \\
\hline \multirow{3}{*}{ Third month } & DGF (-) & 1.77 & 0.77 & 40 & 61.64 & 29.63 & 40 \\
\hline & DGF (+) & 2.37 & 1.14 & 37 & 81.34 & 36.83 & 37 \\
\hline & Total & 2.06 & 1.01 & 77 & 71.10 & 34.52 & 77 \\
\hline \multirow{3}{*}{ End of the first year } & DGF (-) & 1.69 & 0.84 & 35 & 57.40 & 32.51 & 35 \\
\hline & DGF (+) & 2.41 & 1.49 & 32 & 67.53 & 32.73 & 32 \\
\hline & Total & 204 & 1.24 & 67 & 62.24 & 32.76 & 67 \\
\hline
\end{tabular}




\section{Cureus}

\begin{tabular}{|c|c|c|c|c|}
\hline Serum markers & Source of variance & $\mathbf{F}$ & $\mathbf{p}$ & $\eta^{2}$ \\
\hline \multirow{3}{*}{ Creatinine } & Measurement (creatinine) & 2.621 & 0.075 & 0.023 \\
\hline & DGF & 26.950 & 0.0001 & 0.108 \\
\hline & Measurement (creatinine) ${ }^{\star}$ DGF & 2.109 & 0.124 & 0.019 \\
\hline \multirow{3}{*}{ Urea } & Measurement (urea) & 22.000 & 0.0001 & 0.165 \\
\hline & DGF & 17.670 & 0.0001 & 0.074 \\
\hline & Measurement (urea)* DGF & 1.469 & 0.232 & 0.013 \\
\hline
\end{tabular}

TABLE 4: The comparison of the mean serum urea and creatinine levels of the DGF groups according to the measurement at different times (two-factor ANOVA)

DGF: delayed graft function, ANOVA: analysis of variance.

*The interaction of measurements.

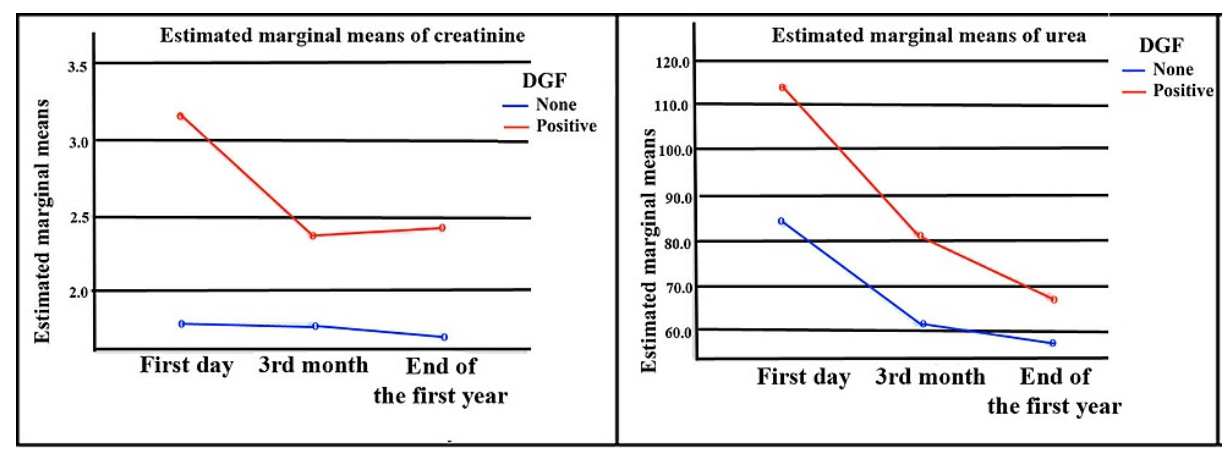

FIGURE 2: Estimated marginal means of serum urea and creatinine levels in both groups

\section{Discussion}

The risk of developing DGF after kidney transplantation from a donation after brain death (DBD) was reported to be between $24 \%$ and $70 \%$ [2]. This rate is higher in kidney transplant patients who have undergone donation after cardiac death $[2,10]$. In our study, people with brain death could become deceased kidney donors. Donations after cardiac death could not be performed because they are not legally possible in our country. DGF was observed in $52.3 \%$ of our kidney transplant recipients. There are many diseases in DGF etiology [1,2]. Cold ischemia time is independently associated with the risk of DGF [10]. HLA mismatch is an independent predictor of DGF [11]. However, the functional amino acid polymorphisms in the antigen recognition region of the HLA-A molecule may have an effect on DGF [12]. Cold ischemia time was higher in DGF (+) group than in DGF (-) group in our study. The groups were found similar concerning HLA mismatch (Table 1).

Delayed graft function is defined as the need for dialysis within the first week after kidney transplantation [2]. During this period, patients are usually taken on dialysis due to life-threatening (e.g., hyperkalemia, metabolic acidosis, pulmonary edema, uremic complications) indications [13]. Serum creatinine alone is not ideal for identifying DGF. However, failure of serum creatinine to fall below pre-transplant levels within one week in patients with DGF can also be seen. There is an insufficient number of studies in the literature investigating the effect of serum urea level on DGF development in kidney transplant recipients [14]. In our study, we found higher serum urea and creatinine levels in DGF (+) patients compared to those with DGF (-).

In addition to measuring blood flow changes in the kidneys, RRI also reflects damage to the tubulointerstitium [7]. Many diseases affecting the tubulointerstitium and some vascular complications are included in the etiology of DGF [1,2]. Among kidney transplant recipients with low RRI values have a lower rate of DGF than those with high values [15]. There are several studies in the literature investigating whether RRI has a predictive role on DGF in kidney transplant recipients $[16,17]$. The elevated RRI is linked to a higher risk of DGF. The predictive efficacy of RRI was higher when the evaluation was done early after 
transplantation [16]. To predict the development of DGF, the sensitivity of RRI in the intraoperative and postoperative first day is higher [17]. However, this application is not easily applied in the clinic. For this reason, we were able to evaluate the RRI value in kidney transplant recipients within the first week (between one and seven days) after transplantation. In long-term follow-up, the patients with lower RRI values showed better graft survival rates than higher ones [15]. Increased RRI values are seen in patients with DGF, acute rejection, and acute tubular necrosis [18]. RRI values better reflect DGF in the first month after kidney transplantation. However, the RRI value between the first and third months could be due to acute changes in the graft [19]. In our study, we found the RRI value to be higher in DGF (+) group than DGF (-) group in the first week after kidney transplantation. In both groups, the value of RRI in the first week was lower than at the end of the first year. Despite these positive results, the two-factor ANOVA analysis found that RRI was insufficient to predict DGF in kidney transplant recipients.

Our study has some limitations. This study was planned retrospectively. Most of the patients in our center had a deceased kidney donor, and the number of patients transplanted from a living donor was low. Thus, subgroups of patients who underwent kidney transplantation from living and deceased donors could not be compared with each other. RRI values of a small number of patients could be measured due to lack of clinical indication and patient incompatibility. Recipient dialysis status, panel reactive antibodies, the detailed information of the donor's kidney, the kidney donor profile index, and warm ischemia time (donor and recipient) could not be determined.

\section{Conclusions}

The value of RRI in the DGF (-) group was lower than the DGF (+) group in the first week after kidney transplantation. The elevated RRI may be linked to a higher risk of DGF within the first week after transplantation. Prospective studies defining the optimal cut-off value and timing measurement of RRI should be designed in this area.

\section{Additional Information \\ Disclosures}

Human subjects: Consent was obtained or waived by all participants in this study. Canakkale Onsekiz Mart University, Clinical Research Ethical Committee issued approval 18920478-050.01.04-E.1900164941. Animal subjects: All authors have confirmed that this study did not involve animal subjects or tissue. Conflicts of interest: In compliance with the ICMJE uniform disclosure form, all authors declare the following: Payment/services info: All authors have declared that no financial support was received from any organization for the submitted work. Financial relationships: All authors have declared that they have no financial relationships at present or within the previous three years with any organizations that might have an interest in the submitted work. Other relationships: All authors have declared that there are no other relationships or activities that could appear to have influenced the submitted work.

\section{References}

1. Yarlagadda SG, Coca SG, Formica RN Jr, Poggio ED, Parikh CR: Association between delayed graft function and allograft and patient survival: a systematic review and meta-analysis. Nephrol Dial Transplant. 2009, 24:1039-47. 10.1093/ndt/gfn667

2. Mallon DH, Summers DM, Bradley JA, Pettigrew GJ: Defining delayed graft function after renal transplantation: simplest is best. Transplantation. 2013, 96:885-9. 10.1097/TP.0b013e3182a19348

3. Butala NM, Reese PP, Doshi MD, Parikh CR: Is delayed graft function causally associated with long-term outcomes after kidney transplantation? Instrumental variable analysis. Transplantation. 2013, 95:1008-14. 10.1097/TP.0b013e3182855544

4. Shoskes DA, Shahed AR, Kim S: Delayed graft function. Influence on outcome and strategies for prevention . Urol Clin North Am. 2001, 28:721-32.

5. Schröppel B, Legendre C: Delayed kidney graft function: from mechanism to translation. Kidney Int. 2014, 86:251-8. 10.1038/ki.2014.18

6. Redfield RR, Scalea JR, Zens TJ, et al.: Predictors and outcomes of delayed graft function after living-donor kidney transplantation. Transpl Int. 2016, 29:81-7. 10.1111/tri.12696

7. Pontremoli R, Viazzi F, Martinoli C, et al.: Increased renal resistive index in patients with essential hypertension: a marker of target organ damage. Nephrol Dial Transplant. 1999, 14:360-5. 10.1093/ndt/14.2.360

8. Lundell A, Persson NH, Källen RR, Ekberg H: Impaired renal artery blood flow at transplantation is correlated to delayed onset of graft function. Transpl Int. 1996, 9(1):57-61. 10.1007/BF00336813

9. Freedland SJ, Mishkin F, Shoskes DA: Effective renal plasma flow calculated from a single blood sample following Technetium-99m mercaptoacetyltriglycine renal scan can predict delayed graft function in renal transplantation. Tech Urol. 2001, 7:281-4.

10. Mannon RB: Delayed graft function: the AKI of kidney transplantation. Nephron. 2018, 140:94-8. $10.1159 / 000491558$

11. Damodaran S, Bullock B, Ekwenna O, Nayebpour M, Koizumi N, Sindhwani P, Ortiz J: Risk factors for delayed graft function and their impact on graft outcomes in live donor kidney transplantation. Int Urol Nephrol. 2021, 53:439-46. 10.1007/s11255-020-02687-5

12. Kamoun M, Holmes JH, Israni AK, et al.: HLA-A amino acid polymorphism and delayed kidney allograft function. Proc Natl Acad Sci U S A. 2008, 105:18883-8. 10.1073/pnas.0810308105 


\section{Cureus}

13. Section 5: dialysis interventions for treatment of AKI . Kidney Int Suppl (2011). 2012, 2:89-115. 10.1038/kisup.2011.35

14. Yarlagadda SG, Coca SG, Garg AX, Doshi M, Poggio E, Marcus RJ, Parikh CR: Marked variation in the definition and diagnosis of delayed graft function: a systematic review. Nephrol Dial Transplant. 2008, 23:2995-3003. 10.1093/ndt/gfn158

15. Tirtayasa PM, Duarsa GW, Situmorang GR, et al.: Association between early resistive index measurement and early graft function and long-term graft survival after kidney transplantation: an evidence-based clinical review. Acta Med Indones. 2019, 51:77-85.

16. Bellos I, Perrea DN, Kontzoglou K: Renal resistive index as a predictive factor of delayed graft function: a meta-analysis. Transplant Rev (Orlando). 2019, 33:145-53. 10.1016/j.trre.2019.03.003

17. Mocny G, Bachul P, Chang ES, Kulig P: The value of Doppler ultrasound in predicting delayed graft function occurrence after kidney transplantation. Folia Med Cracov. 2016, 56:51-62.

18. Chudek J, Kolonko A, Król R, Ziaja J, Cierpka L, Wiecek A: The intrarenal vascular resistance parameters measured by duplex Doppler ultrasound shortly after kidney transplantation in patients with immediate, slow, and delayed graft function. Transplant Proc. 2006, 38:42-5. 10.1016/j.transproceed.2005.12.013

19. de Freminville JB, Vernier LM, Roumy J, et al.: Early changes in renal resistive index and mortality in diabetic and nondiabetic kidney transplant recipients: a cohort study. BMC Nephrol. 2021, 22:62. 10.1186/s12882-021-02263-8 